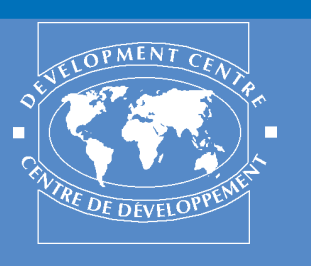

\title{
Repéres
}

n०10

\section{La Dynamique humaine de l'aide}

\author{
par Malcolm MacLachlan et Stuart C. Carr*
}

L'aide internationale au développement apportée par le monde riche aux pays pauvres (« en développement ») représente d'importants flux de capitaux, de ressources humaines et d'assistance technique. Tandis que la direction nette de ces flux reste très controversée, on a identifié plusieurs obstacles à l'utilisation efficiente de l'aide par les pays en développement. Ils tiennent pour beaucoup aux difficultés de mise en oeuvre d'une « bonne gouvernance » (Banque mondiale, 2004). C'est pourquoi les donneurs cherchent de plus en plus à encourager la bonne gouvernance au sein des organismes destinataires de l'aide avec lesquels ils travaillent. Cependant, en sa qualité de directeur exécutif du Fonds mondial, Richard Feachem (2004) note que la question de la bonne gouvernance se pose également, avec une évidence croissante, du « côté de l'offre »: les conflits d'intérêts et la concurrence entre organismes œuvrant dans le cadre des Nations unies et du système d'aide multilatéral, entre pays donneurs et entre une pléthore d'organisations non gouvernementales, provoquent en effet inefficiences et manque de coordination.

Sans faire de l'aptitude à I'organisation une caractéristique du dispositif d'aide, nous pensons que la notion de développement des capacités implique, à l'image du mythe de Sisyphe, qu'on se batte sans cesse contre les mêmes obstacles. Par définition, le développement des capacités dépend de l'apprentissage organisationnel. Nous admettons certes qu'aucun « mode » d'apprentissage particulier ne puisse prétendre avoir la préséance sur les autres, mais nous souhaitons examiner plus en détail le cursus d'apprentissage nécessaire pour que les projets fonctionnent en étant appropriés par les agents. Par-delà les moyens économiques, techniques et politiques nécessaires pour que ces projets marchent, il y a en effet des individus, les destinataires de l'aide, et ce sont ces personnes, qui interagissent avec les systèmes d'aide ou encore qui les représentent, qu'il s'agit de comprendre. Cette dynamique humaine repose sur la motivation, et en particulier sur des aspirations à l'équité sociale. Étudier «comment» ces motivations s'exercent, y compris dans leurs manifestations négatives, est crucial pour le développement des capacités.

\section{Des scénarios récurrents}

\section{Scénario 1 : La double démotivation}

Le premier scénario récurrent a trait au salaire et aux rémunérations (ses variables sont décrites dans la figure 1 ). D'entrée de jeu, les groupes vont comparer leurs rémunérations en termes d'équité (comparaison d'équité). Parmi la catégorie la mieux rémunérée (ex. l'étranger expatrié) peut ainsi naître un certain malaise (ex. la culpabilité), qui se conjuguera à d'autres facteurs de stress pour accélérer un renouvellement des effectifs (départs). Ou encore, les intéressés se mettront à travailler plus dur pour justifier leur meilleur salaire (renforcement des efforts). Mais personne ne pouvant travailler 10 ou 20 fois plus que les autres, ce processus de comparaison aboutira, selon toute probabilité, à une compensation sur un plan psychologique du sentiment d'équité par le raisonnement implicite suivant: " si nous sommes mieux payés, c'est que nous le valons ». Un tel auto satisfecit n'est guère susceptible d'optimiser l'implication dans le travail, et sape 
Figure 1. L'escalade de la double démotivation

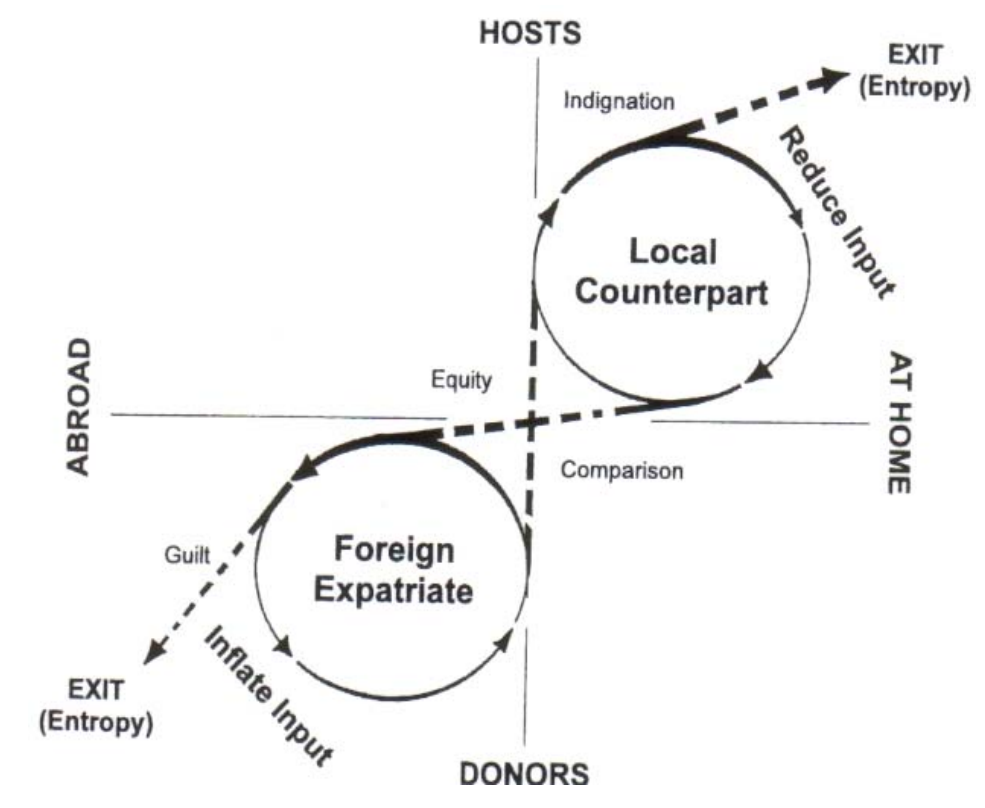

légendes figure 1 :

Hosts $=$ Pays bénéficiaires

Exit (entropy) $=$ Départ (entropie)

Donors $=$ Donneurs

Abroad $=$ À l'étranger

At home $=$ Dans le pays d'origine

subrepticement la motivation (démotivation 1). Cette inflation de l'ego rejaillit sur les catégories moins bien rémunérées, qui la ressentent. Or ces catégories ressentent déjà de l'indignation en raison de leur rémunération inférieure. A défaut de s'en aller (départ pouvant contribuer à une fuite des cerveaux), elles réduiront leurs efforts pour compenser leur sous-rémunération (démotivation 2). Ce fléchissement est alors ressenti à son tour par les catégories mieux payées, les confortant dans la haute opinion qu'elles se sont forgée d'elles-mêmes et justifiant à leurs yeux une nouvelle réduction de leurs propres efforts, et ainsi de suite. Un cercle vicieux se crée. On observe donc, au fil du temps, une double démotivation, à la fois dans le comportement de l'étranger expatrié et dans celui des équipes locales.

La figure 1 fait apparaître les indicateurs psychologiques de la double démotivation : culpabilité et sentiment d'importance dans le groupe des mieux rémunérés; indignation et désimplication pour les personnes moins bien payées. Ces émotions présumées, ainsi que les indicateurs de la double démotivation, ont été vérifiés dans différents contextes, avec plusieurs méthodes de recherche. Nous

\author{
Indignation = Indignation \\ Reduce input $=$ Réduction des efforts \\ Comparison $=$ Comparaison \\ Foreign expatriate $=$ Étranger expatrié \\ Inflate input $=$ Renforcement des efforts \\ Guilt $=$ Culpabilité \\ Equity $=$ Équité
}

observons à chaque fois le même schéma. Le tableau 1 en donne un exemple, avec des données provenant de I'Université du Malawi (UNIMA). Des maîtres de conférence originaires du pays ou venant de l'étranger collaborent à I'UNIMA, moyennant des rémunérations de niveau local pour les premiers, et de niveau international (aide) pour certains des autres.

Il ressort du tableau 1 que les intervenants rémunérés au niveau international se sentent relativement coupables par rapport à leurs homologues locaux ; et ils croient d'autant plus volontiers que les expatriés assurent mieux le travail que leurs collègues locaux (la moyenne reportée au tableau 1 édulcore un certain nombre de réponses plus radicales et, pour raisons de convenance sociale, sousestime aussi probablement le véritable degré d'adhésion aux propositions de l'enquête). Le tableau révèle une donnée fondamentale : les partenaires locaux se font une idée fausse du point de vue des expatriés. Et concernant les propositions de l'enquête concernant les formateurs locaux, les Malawiens sont nettement plus enclins à revendiquer une équité de rémunération, à juger injuste 
Tableau 1. Points de divergences selon les catégories salariales

\begin{tabular}{llc}
\hline & Expatriés & Malawiens \\
\hline $\begin{array}{l}\text { Propositions concernant la motivation au travail des expatriés : } \\
\text { Certains expatriés très bien rémunérés éprouvent de la culpabilité parce qu'ils gagnent } \\
\text { beaucoup plus que le personnel local }\end{array}$ & 3.4 & 2.0 \\
Les expatriés travaillent mieux que leurs homologues locaux & 2.7 & 1.6 \\
Propositions concernant la motivation au travail du personnel local : & 4.1 \\
Les expatriés devraient travailler aux mêmes termes et conditions que le personnel local & 2.2 & 4.6 \\
La majorité des entreprises ne traitent pas leur personnel local de manière équitable & 3.3 & 4.2 \\
Le personnel local est démotivé par la rémunération élevée de certains expatriés & 2.9 & \\
\hline
\end{tabular}

\footnotetext{
Notes : Échelle de 1 à 5 . Plus la note est élevée, plus la personne interrogée approuve la proposition énoncée.

Tous les échantillons comparés sont statistiquement significatifs, après correction de Bonferroni pour l'erreur de type I. Source : Carr et al. (1998).
}

le système de rémunération à deux vitesses, et à considérer que l'important écart de rémunération démotive les personnels locaux. Autre donnée fondamentale: les expatriés se font eux aussi une idée fausse du point de vue de leurs partenaires locaux.

La double démotivation tient au fait que «l'on ne se voit pas comme les autres nous voient ». C'est peut-être l'essence même de «l'apprentissage en simple boucle ». Il convient de noter deux autres aspects importants :

- Dans l'hypothèse où la démotivation du personnel local tiendrait essentiellement à des différences d'attitude induites par des facteurs culturels, les maîtres de conférence expatriés rémunérés sur la base des salaires locaux devraient alors partager le point de vue de leurs collègues rémunérés au niveau international. Or, l'analyse de l'enquête montre que leur opinion s'aligne plutôt sur celle de leurs collègues locaux rémunérés comme eux (Carr et al., 1998). On peut en déduire que c'est le système économique, et non les différences culturelles ou d'attitude, qui crée les conditions d'une déperdition des capacités.

- Un conflit social portant sur un système de rémunération différenciée a fortement perturbé le fonctionnement de l'Université de Papouasie-NouvelleGuinée, qui a dû être temporairement fermée en 1998. Ce cas montre que l'écart de rémunération peut avoir des conséquences allant bien au-delà du mécontentement individuel et provoquer un malaise général à l'échelle de toute une organisation (pour une étude récente de cet aspect, voir Carr, 2004).

\section{Scénario 2 : Payez-moi !}

Dans le scénario 1 , outre les sentiments de disparité liés à la rémunération en soi, la dignité humaine est un autre facteur crucial. La rémunération a tout autant une valeur symbolique que matérielle ou économique : celle de juste salaire pour un travail bien fait. Cette équation espérée engendre, dans le cas de l'aide, divers problèmes que nous appelons la réaction du « Payez-moi ! ». Cette expression « payez-moi! » découle d'anecdotes recueillies sur les réactions locales à l'aide extérieure. Le « folklore » de l'aide voudrait que les populations rechignent, tant qu'on ne les a pas «payées » pour le faire, à par exemple assembler des équipements offerts pour construire des puits antisécheresse, ou à décharger de l'aide alimentaire, à participer à la recherche sur l'éducation ou à assister aux réunions sur la lutte contre le SIDA. Ainsi, une équipe de coopérants est arrivée dans un village rural gravement touché par une sécheresse persistante, dont les habitants devaient parcourir des kilomètres pour trouver de l'eau potable et pour les tâches quotidiennes. L'équipe a demandé aux villageois un coup de main pour décharger des camions les engins de forage, afin de pouvoir creuser sur place un nouveau puits plus profond. À sa grande stupéfaction, les villageois ont déclaré que si les coopérants voulaient leur aide, ils n'avaient qu'à les payer pour ça.

Les visiteurs de protester, arguant que les matériels, tout comme eux-mêmes, n'étaient là pour le bien de la communauté. Les villageois ont rétorqué, en admettant que c'était le cas, qu'il était tout aussi vrai que les coopérants était rémunérés pour ce bon travail. Et que s'ils méritaient d'être payés pour cela, pourquoi pas eux? 
Pour vérifier l'universalité d'une telle réaction en dehors de son contexte particulier, nous avons élaboré un questionnaire à partir d'anecdotes analogues que nous avons transmis à des personnes ayant une expérience de missions d'aide. Elles ont estimé que la réaction du « Payezmoi ! » se serait produite une fois sur cinq dans les anecdotes mentionnées, plutôt que le coup de main bénévole. Cependant la fréquence du «Payez-moi ! » variait selon les cas de figure: plus les populations évoquées avaient été en contact avec des organismes d'aide, plus cette réaction était attendue.

Dans certaines circonstances au moins, il semblerait donc qu'au lieu « d'aider les gens à s'aider eux-mêmes », les projets d'aide suscitent plutôt une réaction de type « payezmoi pour m'aider ». Comment l'expliquer ? On peut souligner l'importance de la dignité et de la réciprocité dans les relations humaines. « Tout don entraîne la perte de quelque chose », telle que la fierté ou le respect de soi. Offrir de temps en temps un cadeau peut relever de la bonté, mais se trouver dans la situation de recevoir constamment des « cadeaux », surtout dans l'incapacité financière de rendre la pareille, peut devenir humiliant, voire susciter du ressentiment. Le moyen de retrouver alors un peu de dignité, en mettant implicitement sur un pied d'égalité sa propre valeur et celle d'autrui, peut consister à demander à être apprécié de la même manière, c'est-àdire en étant payé (rémunéré) pour le temps consacré à quelque chose. Sans compter que dans la pratique, le temps passé à assembler les éléments d'un puits ou à décharger des produits alimentaires pourrait être consacré à d'autres activités, peut-être plus bénéfiques sur le plan personnel. Le «Payez-moi!», comme la double démotivation, peut donc être considéré comme une façon de rétablir l'équité et l'équilibre.

\section{Scénario 3 : La gravité motivationnelle}

Toujours à propos de la dignité, le développement des capacités vise souvent à encourager à mener à bien les réalisations, en reconnaissant donc le caractère motivant de leur accomplissement. La motivation de l'accomplissement revêt au moins deux grandes formes, aussi incontournables l'une que l'autre : I'accomplissement au niveau individuel et l'accomplissement via le groupe social. Ces deux facteurs humains ayant les mêmes traits, il faut, pour favoriser l'accomplissement, préserver un équilibre raisonnable entre ces deux formes de reconnaissance. De même que l'excès de collectivisme étouffe l'individu, l'excès d'individualisme étouffe le groupe, favorisant une réaction collective brutale. Lorsqu'on tente de développer les capacités, l'équilibre (ou du moins la stabilité) de la collectivité est souvent menacé par le «mantra » de l'individualisme et de la promotion personnelle, qui est généralement véhiculé par la culture de la mondialisation. Le même constat vaut pour les systèmes de pouvoir traditionnels, qui privilégient la hiérarchie et l'ancienneté plutôt que l'égalitarisme de mécanismes de gouvernance plus « démocratiques ». Il y a donc ici interaction de deux dimensions essentielles: l'individu par opposition au groupe, et la hiérarchie par opposition à l'égalité.

Ces interactions peuvent exercer une influence spectaculaire sur l'accomplissement et les performances individuelles. Face aux implications individuelles, l'autorité (le patron) peut réagir en tirant vers le haut (attitude encourageante) ou en poussant vers le bas (attitude décourageante). Les collègues réagissent selon un schéma analogue, en poussant vers le haut (attitude encourageante) ou en tirant vers le bas (attitude décourageante). Cette matrice de tensions crée une taxinomie à quatre pôles, un champ de forces sociales qui orientent les relations humaines. Ce champ de forces exerce, ou menace d'exercer, différentes combinaisons de pressions-dépressions sur un individu. C'est une dynamique que nous avons appelée « la gravité motivationnelle » (motivational gravity) (Carr et MacLachlan, 1997). Il importe de préciser que cette « gravité » n'est pas forcément destructrice ou vindicative. Ainsi, tirer un groupe vers le bas a fréquemment pour but de ramener un individu au « milieu », de la même façon que la gravité permet, au sens propre, de garder les pieds sur terre. Par conséquent, la gravité motivationnelle, à l'instar du « Payez-moi ! » et de la double démotivation, rétablit l'équité sociale. C'est une forme de justice réparatrice.

\section{Synthèse}

Le concept de gravité motivationnelle, comme la double motivation et le « Payez-moi !», nous rappelle que les organismes d'aide placent parfois leur personnel local dans la quadrature potentiellement difficile de se sentir tiré vers le haut par l'employeur mais tiré vers le bas (ou ramené au milieu) par les groupes locaux. Nous espérons donc que les notions que nous avons brièvement exposées aideront les partenaires qui œuvrent au développement des capacités, peut-être via des interactions partant de positions personnelles et culturelles différentes, à examiner en détail leur environnement social et politique et à se montrer plus soucieux de retenir des approches durables. Reconnaître le problème, c'est commencer à le résoudre. Dans un monde idéal, nous pourrions définir un large éventail de scénarios récurrents qui nous feraient prendre conscience de la façon dont les autres nous perçoivent, ce qui nous amènerait à réfléchir davantage sur nos pratiques. Cet apprentissage « en double boucle » est une composante clé de la constitution de capacités dans tout groupe ou organisation. En nous appuyant sur cette longue liste, nous devrions, dans l'idéal, synthétiser diverses compétences permettant de surmonter les obstacles déjà identifiés. Les compétences sont spécifiques et d'ordre pratique. Il s'agit de 


\section{Tableau 2. Synthèse thématique des compétences/caractéristiques liées au poste, d'après quatre études d'incidents critiques vécus par des personnes travaillant dans le domaine de l'aide et du développement}

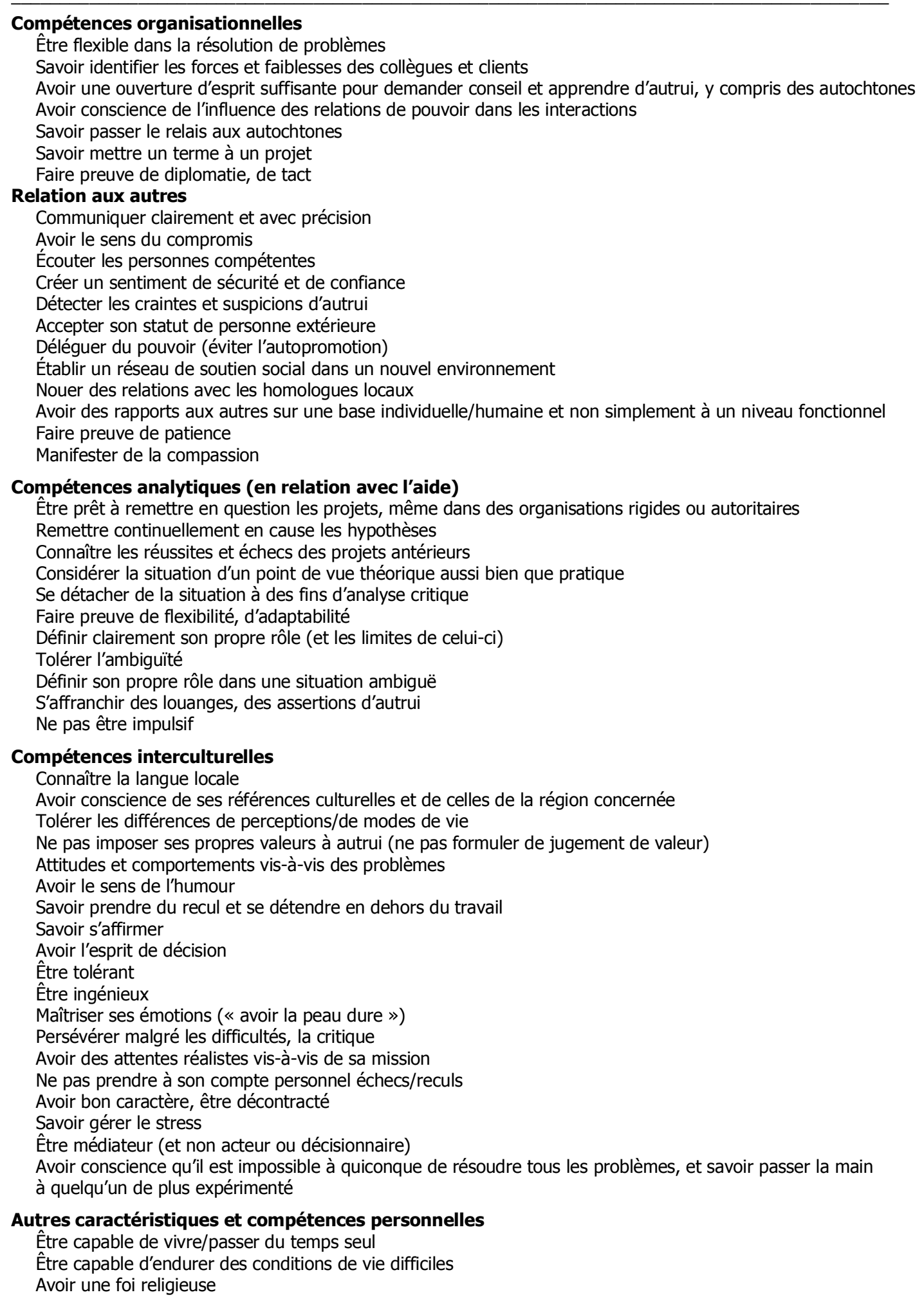

Source: MacLachlan et McAuliffe (2003), reproduit avec l'aimable autorisation des auteurs. 
savoirs pragmatiques, qui reposent sur des connaissances procédurales et non sur des connaissances déclaratives. Concernant le développement des capacités, il est de plus en plus admis que l'aide internationale doit moins être liée à des résultats prédéfinis (réaliser tel projet en tant d'années) qu'à la mise en place des processus spécifiques destinés à étoffer les capacités locales. Tout le monde semble s'accorder sur l'importance des « compétences de processus », dont le développement est, en outre, essentiel à celui des capacités (MacLachlan et Mc Auliffe, 2003).

\section{Compétences de processus}

Dans nos précédentes études des incidents critiques survenant dans le cadre des opérations d'aide internationale, les compétences de processus ont, à plusieurs reprises, été considérées comme les plus importantes, bien plus encore que les compétences techniques. Pour illustrer la méthode d'identification des compétences liées aux postes, et pour préciser les caractéristiques des incidents de parcours, voici le résumé de l'un de ces incidents, relaté par une professeur de nutrition d'un lycée des Îles Salomon, qui le considère comme un incident critique positif (Cullinan et MacLachlan, 2004).

Après s'être informée autant que possible sur les habitudes alimentaires indigènes, ce professeur a identifié une carence en protéines comme principal déséquilibre du régime alimentaire local. Selon elle, la population avait besoin d'une source de protéines autre que le poisson et tout aussi acceptable. Le chef d'établissement (un expatrié) était persuadé que le lait apportait la solution et refusait d'écouter l'enseignante lorsqu'elle s'efforçait de lui faire comprendre qu'il était tout simplement inconcevable pour les autochtones de boire du lait : elle les avait interrogés sur ce point. Les gens n'avaient jamais bu de lait et cette idée leur répugnait. L'enseignante pressentait que de nombreux projets avaient échoué par méconnaissance des personnes qu'ils prétendaient aider. Elle voulut lancer un programme d'élevage de poulets, en se heurtant à la résistance du directeur à chaque étape. Lorsqu'elle réussit enfin à introduire quelques volailles dans l'école, pour apprendre aux élèves comment s'en occuper et les cuisiner, le directeur a tenu à ce qu'elles soient vendus. Elle se souvient d'une discussion longue et animée avec le chef d'établissement pour le convaincre de la laisser donner aux élèves quelques poulets à rapporter chez eux pendant les vacances de Noël. Elle a su le persuader. Ce programme a remporté un vif succès. À la rentrée, les élèves savaient élever des poulets et l'avaient appris à leurs familles. Et dès qu'ils ont pris goût au poulet, les gens l'ont adoré.

Cet incident est qualifié de positif car l'enseignante a su atteindre son objectif, c'est-à-dire identifier, pour le régime alimentaire local, une nouvelle source de protéines qui soit acceptable et durable. Prenant cet incident pour exemple du type de travail que l'on attendait d'elle dans le cadre de sa mission, cette personne a mis en exergue un certain nombre de compétences/caractéristiques pertinentes: connaître la culture locale (se renseigner sur la nourriture locale et les aliments incompatibles avec les traditions), savoir écouter (interroger les autochtones sur leur régime alimentaire et leurs préférences avant de lancer un projet), persévérer malgré les obstacles (poursuivre en dépit de I'opposition du chef d'établissement). Si l'on en revient aux scénarios collectifs décrits plus haut, il s'agit de faire preuve de respect et d'apporter de la dignité. Dans la même perspective, les chercheurs ont mis en avant les compétences suivantes : savoir communiquer (atteindre son objectif à force de discussions/persuasion auprès du directeur), faire montre de flexibilité dans la résolution d'un problème malgré de maigres moyens (reconnaître que bien d'autres options n'étaient pas viables, culturellement ou financièrement; trouver la solution de l'élevage de poulets), ne pas imposer ses propres valeurs à autrui (admettre qu'une solution qui semblait évidente au directeur était inacceptable pour des personnes d'une autre culture, dans un autre contexte), connaître les réussites ou les échecs des projets précédents (savoir que des programmes de nutrition antérieurs avaient échoué pour n'avoir pas tenu compte des besoins/souhaits de la population locale).

\section{Études comparatives des compétences de processus}

Le tableau 2 synthétise les conclusions de plusieurs études (MacLachlan et McAuliffe, 2003) et les regroupe en catégories thématiques. Nous estimons que si certaines de ces compétences sont, de toute évidence, « universellement » requises, d'autres sont plus particulièrement nécessaires dans les situations dans lesquelles se retrouvent les équipes d'aide, par exemple dans les scénarios que nous avons évoqués précédemment. C'est sur ces points que nous centrons notre analyse.

En termes de compétences organisationnelles par exemple, on trouve parmi les caractéristiques fondamentales liées au poste la conscience de la relation de pouvoir implicite entre l'expatrié (le donneur) et l'autochtone (le bénéficiaire), conjuguée peut-être à une ouverture d'esprit affichée pour apprendre d'autrui et à la disponibilité à passer le relais aux intervenants locaux. S'agissant de la relation personnelle avec les autres, les compétences de processus essentielles sont l'aptitude à créer un sentiment de sécurité et de confiance, la capacité à déceler les suspicions et les peurs d'autrui, à accepter son statut de personne extérieure et à déléguer du pouvoir, ainsi qu'à manifester de la compassion.

Les compétences interculturelles sont peut-être les plus fortement liées au contexte des missions d'aide, et celles que nous avons identifiées dans nos travaux (conscience de ses perspectives propres et de celles des autochtones, tolérance en général, absence de jugements de valeur) exigent toutes, au moment des décisions, une capacité à 
s'abstenir de se considérer comme la référence centrale. Cette aptitude importe également pour prendre du recul par rapport à un projet et utiliser des compétences analytiques, pour remettre en question les objectifs et les hypothèses des projets, pour évaluer son propre rôle et ses limites, et pour gérer l'ambiguïté, parfois indépendamment du soutien des autres. Enfin, les attitudes/ comportements décrivent un certain nombre de caractéristiques sans doute intimement liées à la personnalité, telles la capacité à prendre du recul et de se détendre en dehors du travail, la maîtrise de ses émotions et la tolérance. De même, dans nos recherches sur la double démotivation, des qualités personnelles telles que savoir assumer un écart de rémunération atténuent la démotivation individuelle (McLoughlin et Carr, 1997 ; pour d'autres exemples de traits de personnalité saillants, voir MacLachlan et Carr, 1999).

Au vu des nombreux incidents que nous avons étudiés, il est clair que ce n'est pas le travail lui-même que les individus trouvent le plus éprouvant, mais le contexte inter et intra personnel dans lequel il s'exerce. Pour déterminer dans quelle mesure une personne sera efficace dans son travail, il est crucial de savoir comment elle gère ses relations avec les autres et comment elle gère « mentalement » les situations (en cas de catastrophes naturelles, de privations durables, de conflits affectant des enfants, etc.). Le plus souvent, les tensions interpersonnelles auxquelles nous avons fait allusion n'impliquent pas simplement la relation aux clients, mais également (et peutêtre surtout) la relation à la « politique organisationnelle » dans et entre les organismes.

Nous nous sommes appuyés sur des exemples d'incidents critiques pour illustrer « comment » identifier certaines des compétences de processus, essentielles dans la constitution de capacités significatives. Auparavant, nous avons également décrit trois scénarios collectifs axés sur I'analyse des interactions de groupe et sur la manière dont l'inégalité et l'injustice ressenties peuvent entraver les progrès. Ayant affirmé que ces deux types de connaissance se chevauchent et peuvent contribuer à l'apprentissage organisationnel qui intervient dans le champ de l'aide internationale, nous terminons par l'étude des systèmes à mettre en place pour que les organisations puissent effectivement procéder à cet apprentissage.

\section{Une infrastructure pour apprendre}

Dans de nombreux organismes d'aide, en raison du foisonnement des activités, il est souvent impossible « d'enregistrer » les enseignements d'une mission ou d'un projet, parce qu'il faut réagir rapidement à de nouvelles demandes et opportunités. Cependant, nous avançons que le donneur fera preuve de bonne gouvernance s'il parvient en priorité à tirer des leçons de sa propre expérience. Pour ce faire, I'organisation doit créer un système comportant certaines étapes fondamentales :

i) Consigner ses activités en cours et antérieures Enregistrement.

ii) Disposer d'un moyen de faire systématiquement le point sur les expériences de son personnel - debriefing.

iii) Avoir un moyen d'accéder aisément et rapidement à des données pertinentes pour d'autres projets - Accès.

iv) Extraire ces données sous un format mettant en avant la manière dont l'organisation peut tirer profit d'expériences passées et mettre ce savoir au service de nouvelles initiatives - Transfert.

Si la plupart des organisations essaient, de manière informelle, d'introduire un cycle d'apprentissage s'apparentant à ce schéma, l'expérience quotidienne atteste de la difficulté, pour les nouvelles actions, à reprendre là où les précédentes se sont arrêtées. Pour que ce type d'apprentissage « en double boucle » puisse se concrétiser, le processus décrit doit être institutionnalisé, et l'organisation doit être claire sur ce point. Certes, le développement de systèmes de TIC (technologies de l'information et de la communication) et de protocoles standard pour le debriefing et la formation à leur usage accroît indubitablement la charge administrative qui pèse sur une organisation ; il reste malgré tout un investissement stratégique qui promet d'être fructueux à court terme (pour les projets à décider par I'organisation), ainsi qu'à plus long terme, via l'autonomisation et le développement des capacités.

Il peut être utile de donner un exemple de I'utilisation immédiate de ces systèmes. Les compétences identifiées lors des debriefings d'incidents critiques apportent l'expérience de terrain permettant de former sans attendre les personnes devant mener des missions dans des pays en développement. Il ne s'agit donc pas simplement de compétences et caractéristiques abstraites. Les incidents critiques peuvent être perçus comme une source de savoir, inspirer un jeu de rôle, et les compétences et caractéristiques requises peuvent être définies avant que les chargés de mission ne se rendent sur le terrain. C'est donc au tout début de l'élaboration des opérations de développement, avant même le départ des chargés de mission, qu'il convient de développer les compétences de processus et de les améliorer progressivement, grâce au retour d'information sur l'expérience concrète acquise lors d'incidents critiques réels et à la prise en compte de leur complexité contextuelle et des frustrations qui les accompagnent.

Un argument symétrique s'applique à « l'autre face » des partenariats de développement, à savoir aux bénéficiaires des projets d'aide. Ce qui est vraiment nécessaire, c'est une analyse mutuelle des écarts. En effet, la population locale a rarement l'occasion de recevoir une formation préalable à l'arrivée d'une mission, analogue à celle des préparatifs de la mission. Ainsi, pour prévenir les scénarios de double démotivation, chaque groupe impliqué pourrait avoir l'opportunité de comprendre les incidents critiques 
antérieurs et les leçons qu'il peut en tirer. Ce type d'apprentissage est aussi complexe et difficile qu'utile. Il n'est pas seulement souhaitable ; il doit intégrer un système d'évaluation de ce qu'on fait, en rapport avec les autres. Il n'y a sans doute pas d'enjeu plus « critique », dans la vie d'une organisation, que des partenariats axés sur le

\section{Implications pour l'élaboration}

de politiques

\section{développement des capacités.}

L'accent que nous avons mis sur la dynamique humaine de I'aide internationale comporte d'importantes implications pour l'élaboration de politiques. Tout d'abord, la mise en œuvre des politiques est entravée par l'incapacité à prendre en compte la dynamique humaine locale, et notamment la manière dont les personnes chercheront à protéger leur estime de soi et leur identité sociale. L'influence des facteurs pécuniaires sur l'environnement motivationnel en général, le sens de l'équité et de la justice des populations doivent être pris en considération, de même que les facteurs de réceptivité qui naissent des différences de contexte social - une approche « unilatérale » ne saurait convenir à toutes les situations. Deuxièmement, I'apprentissage organisationnel ne peut être mis en œuvre par une poignée d'individus éclairés. Une organisation doit répondre à ce besoin par une approche systémique qui mobilise l'expérience de tous pour contribuer à son capital intellectuel, à celui des organisations partenaires et à celui d'autres éventuels partenariats de développement des capacités. Troisièmement, si certaines organisations répugnent à communiquer, l'intérêt général sera vraisemblablement mieux servi si les fonds publics sont dirigés vers les organisations désireuses de partager leur savoir avec d'autres. Il en ressort que l'apprentissage organisationnel n'est pas seulement de nature « verticale », au sein des organismes d'aide, mais également « horizontale », entre donneurs et organisations partenaires, et vice versa.

Les actions de développement international sont traditionnellement centrées sur les moyens matériels et la compétence technique nécessaire pour utiliser ces ressources. Plus récemment, on a commencé à s'écarter de cette « assistance technique » pour adopter une nouvelle philosophie : faciliter le développement en améliorant les capacités locales. Pour concrétiser cette approche, il faut non seulement étudier de près et améliorer la gouvernance des pays destinataires, leurs structures et institutions, mais aussi améliorer la gouvernance des donneurs. Dans ce dernier cas, il est crucial d'instaurer des systèmes de ressources humaines aptes à contribuer au développement des capacités, par exemple via l'apprentissage à partir des expériences antérieures de l'organisation. Que ce soit au niveau de chaque intervenant sur le terrain ou des interactions au sein du groupe ou de l'organisation, I'apprentissage organisationnel dans les organismes d'aide est un prérequis fondamental, voire un impératif moral si I'on veut investir sur la base des améliorations de la gouvernance locale. 


\section{Remerciements}

Cette étude s'appuie sur les travaux d'un séminaire qui s'est déroulé au siège de l'OCDE le 29 avril 2004. Nous remercions pour leurs commentaires Eilish McAuliffe, Karen Cheng, Charles Hongoro, Jonathan Sibley, Colleen McFarlane, Frank O'Connor, Leo Marai, Ishbel McWha et Dianne Gardner. Nous adressons également nos remerciements à la School of Psychology (Massey University), ainsi qu'au Trinity College Dublin, pour leur soutien constant.

\section{Bibliographie}

Carr, S.C. (2004), Globalisation and Culture at Work: Exploring their Combined Glocality, Kluwer, Boston, MA.

Carr, S.C. et M. MacLachlan (1997), "Motivational Gravity" in D. Munro, J.F. Schumaker et S.C. Carr (sous la direction de), Motivation and Culture (pp. 133-155), Routledge, New York.

Carr, S.C., E. McAuliffe et M. MacLachlan (1998), Psychology of Aid, Routledge, Londres.

Cullinan, S. et M. MacLachlan (2004), "Critical Incidents for Irish International Development Workers: Building on Experience", Administration, 51, pp. 87-109.

Feachem, R. (2004), "Overview of the Global Fund and its Financing Activities", document présenté lors du séminaire intitulé New Global Initiatives to Fight the HIV/AIDS Epidemic: Challenges and Opportunities, Royal Irish Academy, Dublin, 24 février.

MacLachlan, M. et E. McAuliffe (2003), "Poverty \& Process Skills", in S.C. Carr et T.S. Sloan (sous la direction de) Poverty \& Psychology: From Global Perspective To Local Practice, Kluwer, New York.

MacLachlan, M. et S.C. Carr (1999), "The selection of international assignees for development work", Irish Journal of Psychology, 20, pp. 39-57.

McLoughlin, D. et S.C. Carr (1997), "Equity Sensitivity and Double De-motivation", Journal of Social Psychology, 137, pp. 668-670.

Banque mondiale (2004), Rapport sur le développement dans le monde 2004 : Mettre les services de base à la portée des pauvres, Washington, D.C.

\begin{abstract}
* Le professeur Malcolm MacLachlan travaille dans le « Centre for Global Health » et dans le « Department of Psychology » du « Trinity College Dublin » (Irlande). Il a suivi une formation en psychologie clinique, discipline dans laquelle il a exercé avant de se tourner vers le conseil en gestion des facteurs humains, au Royaume-Uni. Maître de conférence à la « University of Malawi », il est également professeur invité à la «University of Limpopo » et à celle du Cap (Afrique du Sud). Ses principaux axes de recherche sont l'apprentissage organisationnel et les facteurs humains dans l'aide internationale, les aspects culturels de la santé et du développement, ainsi que les aspects psycho-sociaux du handicap physique.
\end{abstract}

* Le professeur Stuart Carr dirige le «Poverty Research Group », basé à la « Massey University » (Nouvelle Zélande). Il y coordonne le programme de psychologie du travail et des organisations. Après avoir obtenu un PhD en évolution sociale, Stuart Carr a travaillé à la « University of Malawi », à celle de Newcastle (Australie), ainsi que dans le Territoire du Nord (Australie). Il est également professeur invité à I'« Universitas Indonesia » (Indonésie), à la « Srinakharinwirot University «(Thaïlande) et auprès de I'Unesco. Son principal axe de recherche est I'application de la psychologie du travail et des organisations à la lutte contre la pauvreté, à travers la responsabilité sociale de l'entreprise, la dynamique d'équipe et la mobilité internationale. 
Pour consulter les Repères du Centre de développement

www.oecd.org/dev/reperes

\section{les Cahiers de politique économique}

www.oecd.org/dev/cahiers

ou les nouveaux Documents de travail

www.oecd.org/dev/dt

OCDE $\ll O$

Les lecteurs sont invités à citer ou reproduire les informations des Repères du Centre de développement de I'OCDE dans leurs propres publications. En échange, le Centre demande les remerciements de rigueur ainsi qu'un exemplaire de la publication. Le texte intégral des Repères et d'autres informations sur le Centre de développement et ses travaux sont disponibles sur : www.oecd.org/dev.
Centre de développement de I'OCDE

2, rue André-Pascal, 75775 Paris Cedex 16, France Tél : $33(0) 145.24 .82 .00$ Fax : 33 (0)1 44.30.61.49 mél : cendev.contact@oecd.org 